\title{
Deformable gas-filled microbubbles targeted to P-selectin
}

\author{
Joshua J. Rychak ${ }^{\mathrm{b}}$, Jonathan R. Lindner ${ }^{\mathrm{a}}$, Klaus Ley ${ }^{\mathrm{b}, \mathrm{c}}$, Alexander L. Klibanov ${ }^{\mathrm{a}, \mathrm{b}, \mathrm{c}, *}$ \\ ${ }^{a}$ Cardiovascular Division, University of Virginia, Charlottesville, VA 22908-0158, USA \\ ${ }^{\mathrm{b}}$ Department of Biomedical Engineering, University of Virginia, Charlottesville, VA 22908-0158, USA \\ ${ }^{c}$ Cardiovascular Research Center, University of Virginia, Charlottesville, VA 22908-0158, USA
}

Received 19 December 2005; accepted 9 June 2006

Available online 14 June 2006

\begin{abstract}
Ultrasound contrast microbubbles have been successfully targeted to a number of intravascular disease markers. We hypothesized that targeted delivery could be improved further, by making the microbubbles deformable, leading to increased microbubble-endothelium adhesion contact area and stabilized adhesion. Activated leukocytes utilize such strategy; they deform after binding to inflamed endothelium in the vasculature. Lipid-shell microbubbles were targeted to the endothelial inflammatory protein P-selectin with a monoclonal anti-P-selectin antibody attached to the microbubble shell. Deformable microbubbles were created by controlled pressurization with partial gas loss, which generated an average excess shell surface area of $\sim 30 \%$ and the formation of outward-projected wrinkles and folds. Targeted microbubble adhesion and deformability were assessed in the parallel plate flow chamber under shear flow. Sustained adhesion of deformable microbubbles at wall shear stresses between 0.4 and $1.35 \mathrm{dyn} / \mathrm{cm}^{2}$ was consistently better than adhesion of wrinkle-free microbubbles. Over this shear range, targeted wrinkled microbubbles were deformed by shear flow, unlike wrinkle-free microbubbles. In a murine cremaster inflammation model, a significant improvement of deformable microbubble targeting was observed by intravital microscopy. Overall, the mechanical aspects of adhesion, such as particle shape, deformability and surface microstructure, are important in engineering efficient site-targeted particle-based agents for medical imaging and therapy.
\end{abstract}

(C) 2006 Elsevier B.V. All rights reserved.

Keywords: Targeting; Targeted imaging; Adhesion; Ultrasound contrast; Microbubbles

\section{Introduction}

Specific molecular responses on the vascular endothelium are common in many disease states. Many of the specific molecular components upregulated on the surface of vascular endothelium due to a variety of pathologies such as atherosclerosis, ischemiareperfusion injury, and inflammation have been identified. However, clinical methods of non-invasive diagnosis, assessment and imaging of the early states of the vascular response to underlying pathologies are still limited. Recently, targeted contrastenhanced ultrasound has shown potential for identifying the specific endothelial markers of disease (for review, see [1]). This method relies upon the targeted adhesion and ultrasound imaging

\footnotetext{
* Corresponding author. Cardiovascular Division, University of Virginia, Charlottesville, VA 22908-0158, USA. Tel.: +1 434243 9773; fax: +1 434982 3183.

E-mail address: ALK6N@virginia.edu (A.L. Klibanov).
}

of intravascular ultrasound contrast agents on the vascular endothelium in regions of pathology.

Ultrasound contrast agents are typically gas microbubbles encapsulated by a protein, polymer, or lipid shell (for review, see [2]). Targeted microbubbles are created by attaching a targeting ligand (such as a monoclonal antibody or a peptide) specific for the desired (endothelial) marker onto the shell of the microbubbles. The microbubbles are injected intravenously, accumulate at the intended target site after a short circulation period, and are typically cleared from the bloodstream within a fraction of an hour. Upon examination with an ultrasound medical imaging system, the adherent microbubbles generate an acoustic response signal that is displayed as video enhancement of the target tissue on the system screen. Targeted ultrasound contrast agents have been used to assess vascular pathology associated with several intravascular markers, including P-selectin [3], ICAM-1 $[4,5]$, GpIIb/IIIa [6,7], the $\alpha_{v}$ integrins [8,9] and other markers of tumor angiogenesis [10]. Successful targeted contrast ultrasound imaging in these systems is possible in part due to extremely 
high microbubble detection sensitivity of ultrasound imaging, with the capability to visualize individual microbubbles [11]. Microbubbles have also shown promise as vehicles for ultrasound-aided drug $[12,13]$ and gene $[14,15]$ delivery. However, the efficiency of the microbubble-target interaction may be low under physiologically relevant flow conditions.

The adhesion of microbubbles targeted to P-selectin using an anti-P-selectin monoclonal antibody $(\mathrm{mAb})$ has been previously investigated in vitro [16] using the inverted parallel plate flow chamber in a flow-through mode. This study revealed that microbubble retention at the target from the flowing media decreased to nearly undetectable levels above a moderate wall shear stress of $\sim 1.5 \mathrm{dyn} / \mathrm{cm}^{2}$. Additionally, microbubble retention was shown to be dependent upon the target site density. This behavior may impair the ability of targeted contrast-enhanced ultrasound to detect pathology in high shear stress blood vessels. We hypothesized that the ability of these microbubbles to achieve stable adhesion may be enhanced by increasing the number of microbubble-target bonds.

Many biological systems exhibit very efficient adhesion, and leukocyte adhesion to inflamed vascular endothelium is particularly robust. This process, described by a series of steps known as the leukocyte adhesion cascade [17], is analogous to the targeted retention of microbubbles desired for efficacious contrast-enhanced ultrasound imaging. Leukocyte activation induces cytoskeletal re-arrangements that results in increased deformability. This active increase in deformability has been shown to be partially responsible for effective leukocyte adhesion to endothelium, which depends upon fluid forces that cause a passive redistribution of the plasma membrane $[18,19,20]$. This results in characteristically teardrop-shaped leukocytes, which were observed in early intravital studies [21]. Leukocyte deformation correlates with the fluid force acting upon it $[18,22]$. It has been hypothesized that this deformation enhances a leukocyte's state of adhesion by increasing the leukocyteendothelium contact area, thus augmenting the potential number of adhesive bonds $[22,23,24]$. The ability to deform may further stabilize adhesion by diminishing the profile of the attached leukocyte in the shear field, thus reducing the dislodging force on the adherent cell [25]. Additionally, binding through outward-projected microvilli may stabilize adhesion by altering the fluid force distribution upon the adhesive bonds. For example, projection of the adhesive ligand away from the body of the cell decreases the force on the rearmost load-bearing bond [26,27], and tethering through extensible microvilli may further decrease the stress on the bond between the targeting ligand and receptor $[28,29]$.

Many of the above-mentioned deformation-related characteristics that have been shown to enhance leukocyte adhesion in shear flow are passive biophysical processes that may be engineered into inactive targeted particle. The purpose of the current study was to determine whether microbubbles engineered to possess an enhanced ability to deform demonstrate an improvement of targeted adhesion. Several previous studies have explored the ability of the appropriate leukocyte adhesion molecules to mediate targeted delivery [30,31,32]; however, none have investigated the sophisticated mechanical strategies used by leukocytes. Spherical microbubbles might have difficulty to deform under physiological shear flow conditions and, thus, exhibit infrequent or unstable adhesion. A simple method of altering targeted microbubbles to generate excess shell surface area, which grants microbubbles an enhanced ability to deform when adherent in shear flow was proposed for this study. The excess shell of these deformable microbubbles was manifested as micrometer-scale outward-projected folds and wrinkles, and we refer to this formulation as wrinkled microbubbles. The adhesive properties of wrinkled microbubbles and the parent spherical microbubbles targeted to P-selectin were analyzed in shear flow in vitro and in vivo. The sustained adhesion of wrinkled microbubbles was significantly greater than that of spherical microbubbles over a range of target site densities and wall shear stresses, and the enhancement of adhesion of wrinkled microbubbles correlated positively with an increased microbubble deformation. This study presents evidence that one of the mechanical strategies utilized by leukocytes to enhance adhesion under flow may be applied to the delivery of targeted intravascular agents. This approach should be generally applicable to the future designs of micro- and nanopharmaceutical devices.

\section{Materials and methods}

\subsection{Antibodies and protein substrates}

The rat monoclonal antibody against murine P-selectin Rb40.34 [33] was purified from hybridoma supernatant at the University of Virginia Lymphocyte Culture Center and dialyzed overnight against Dulbecco's phosphate-buffered saline (DPBS) using a 10,000-Da cut-off dialysis cartridge. Rb40.34 was biotinylated via a standard protocol with $N$-hydroxysuccinimidobiotin with a coupling efficiency of approximately 0.4 mol biotin per mole of Rb40.34 as assessed using a standard fluorometric 2-(4'-hydroxyazobenzene)benzoic acid (HABA) assay $[16,34]$. FITC-conjugated goat anti-mouse $\operatorname{IgG}$ secondary antibody was used for probing microbubbles for the presence of primary antibody. The murine P-selectin $\mathrm{Fc}$ fusion protein (R\&D Systems, Minneapolis, MN) and casein blocker solution were used for the target plate coating and nonspecific interaction blocking, respectively.

\subsection{Targeted microbubble preparation}

Microbubbles used in these experiments were composed of a decafluorobutane $\left(\mathrm{C}_{4} \mathrm{~F}_{10}\right.$, Flura Corp., Newport, $\left.\mathrm{TN}\right)$ gas core encapsulated by a lipid monolayer shell consisting mainly of distearoylphosphatidylcholine. A brush of poly(ethylene glycol) (PEG) was grafted onto the lipid shell. Microbubbles containing biotin at the distal tip of the PEG brush were prepared as described previously [35]. Briefly, $\mathrm{C}_{4} \mathrm{~F}_{10}$ gas was dispersed by probe-type sonication into an aqueous micellar solution of distearoylphosphatidylcholine, PEG-40 stearate and biotin-PEG77-distearoylphosphatidylethanolamine. Fluorescent microbubbles were prepared by incorporating a small amount of lipid fluorescent probes (DiO or DiI) (Molecular Probes, Eugene, $\mathrm{OR}$ ) into the lipid mixture. Biotin-conjugated microbubbles were 
stored at $4{ }^{\circ} \mathrm{C}$ for up to 2 months, with slight (less than $13 \%$ ) gas loss as determined by microscopy observations and particle size distribution analysis with a Coulter Multisizer IIe counter (Beckman Coulter, Boca Raton, FL).

Microbubbles were targeted to P-selectin by attaching biotinylated Rb40.34 to the biotin-PEG-lipid anchor with a streptavidin link [3]. Biotin conjugated microbubbles were washed with degassed DPBS five times in a bucket rotor centrifuge at $\sim 30 \times g$ for 4 min to remove excess free unincorporated lipid from the microbubbles, and $3 \mu \mathrm{g}$ of streptavidin per $10^{7}$ microbubbles were added to the washed dispersion. Following 30 min of incubation on ice, the microbubbles were washed twice to remove unreacted streptavidin, and incubated on ice with $7.5 \mu \mathrm{g}$ of biotinylated antibody Rb40.34 for $30 \mathrm{~min}$ and subsequently washed to remove free antibody.

\subsection{Preparation of wrinkled microbubbles}

Wrinkled microbubbles were created by pressurizing the preparation described above. Two milliliters of the microbubble dispersion was drawn into a $10 \mathrm{ml}$ syringe containing $8.0 \mathrm{ml}$ of air. A stopcock valve attached to the syringe was closed and plunger depressed such that a hydrostatic pressure of $750 \mathrm{~mm}$ $\mathrm{Hg}$ was applied to the dispersion for $30 \mathrm{~s}$. The applied pressure was measured with an electronic manometer attached to the stopcock. After pressurization, the microbubble dispersion was washed three times to eliminate completely crushed microbubbles from the preparation. The wrinkled microbubbles were then conjugated to streptavidin and Rb40.34, as described above. To enable visualization of surface features and to discriminate wrinkled from spherical microbubbles during experiments, DiI was used to label the wrinkled microbubbles. Wrinkled microbubbles were used within $4 \mathrm{~h}$ of preparation, and no gas loss or re-inflation of the shell was detected during this period by microscopic examination and Coulter Multisizer analysis. The Coulter measures the change in electrical impedance as a particle enters a sensing aperture and passes between a set of electrodes, a procedure known as electrozone sensing. A change in impedance is proportional to the volume of the particle between the electrodes, and the Coulter measurements reflect primarily the volume of the gaseous core.

\subsection{Flow cytometry}

Flow cytometry was performed to verify the presence of equivalent quantities of $\mathrm{Rb} 40.34$ on the surface of wrinkled and spherical microbubbles. The microbubbles were subjected to repeated centrifugal flotation until the size distributions of both populations were comparable (difference of less than $1 \%$ ), as assessed by Coulter Multisizer analysis of the respective mean and median diameters. Preparations of $5 \times 10^{7}$ wrinkled or spherical microbubbles were conjugated to the targeting ligand $\mathrm{Rb} 40.34$ as described above. Targeted microbubbles $\left(10^{7}\right.$ particles) were incubated with $3.7 \mu \mathrm{g}$ FITC-conjugated goatanti mouse $\mathrm{IgG}$ secondary antibody for $30 \mathrm{~min}$ and subsequently washed 3 times. $10^{5}$ microbubbles per population were analyzed on a FACSCalibur (Becton-Dickinson, Franklin Lakes, $\mathrm{NJ}$ ) flow cytometer, using an excitation wavelength of $488 \mathrm{~nm}$
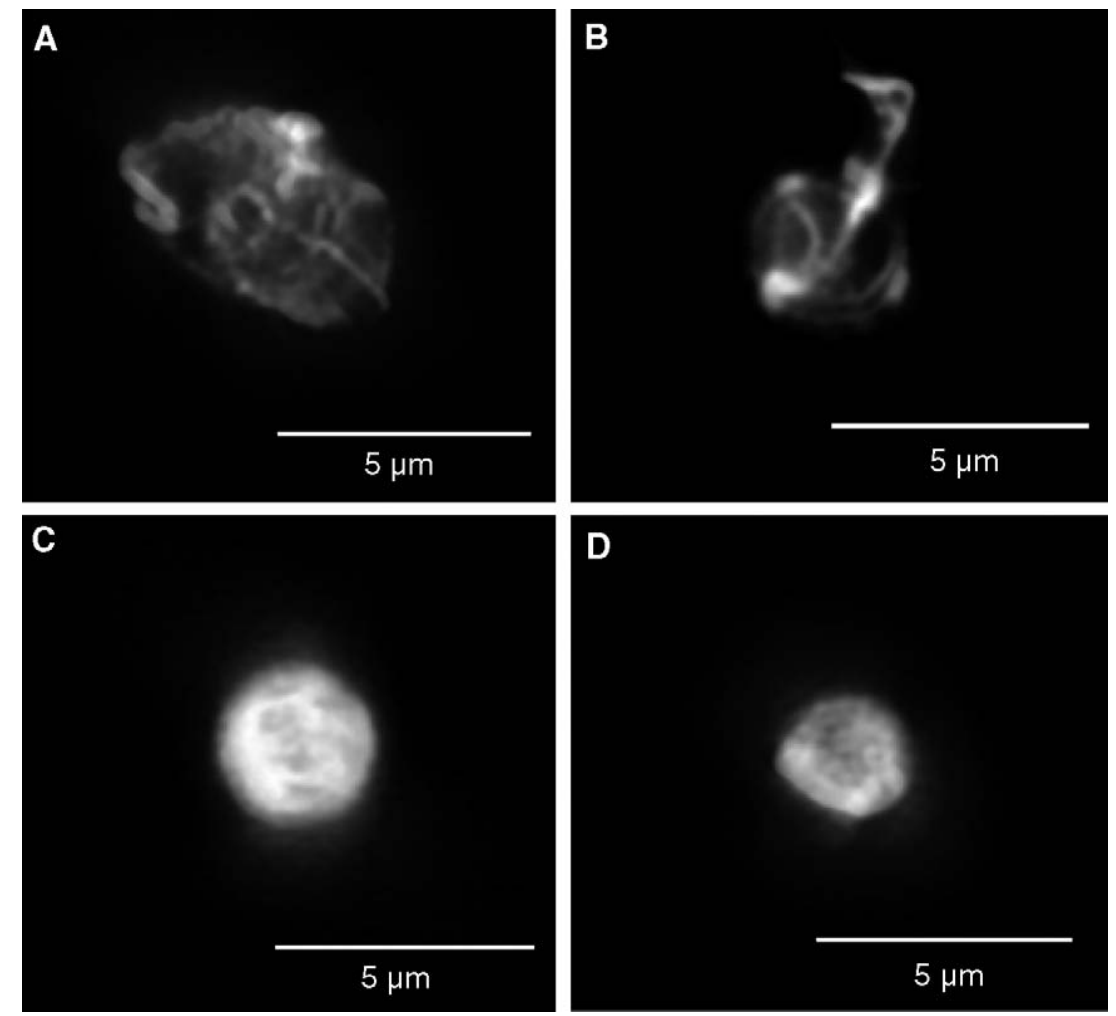

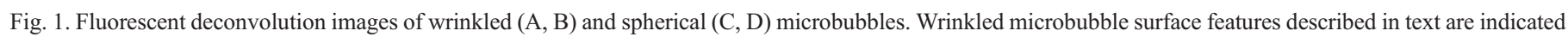
by arrows. Scale bar is $5 \mu \mathrm{m}$. 
and $530 \mathrm{~nm}$ emission. Data were analyzed using FloJo (Tree Star, Ashland, OR).

\subsection{Fluorescence deconvolution microscopy}

Fluorescent images of wrinkled and spherical microbubbles were taken on a DeltaVision (Applied Precision, Issaquah, WA) inverted microscope. Biotinylated, DiI-labeled wrinkled or spherical microbubbles were bound to avidin-coated glass coverslips and kept immersed in degassed DPBS at all times. Wide-field optical sections were acquired with a 1.4 NA 100X oil immersion objective. The optical sections were deconvolved with an experimentally determined three-dimensional point spread function to obtain the final images. Images were presented as maximum intensity volume projections, computed using the DeltaVision software package [36].

\subsection{P-selectin target preparation}

Thirty-five millimeter polystyrene dishes (Corning) were washed with methanol and air-dried prior to adsorption of Pselectin. 25, 150, or $250 \mathrm{ng}$ of recombinant P-selectin were diluted to a final volume of $200 \mu \mathrm{l}$ in DPBS and applied to a $1 \mathrm{~cm}$ circle at the center of the dish. The dishes were then sealed inside a plastic bag to prevent excess evaporation and incubated overnight at $4{ }^{\circ} \mathrm{C}$. Dishes were subsequently washed five times with $0.05 \%$ Tween-20 in DPBS and blocked with $2.0 \mathrm{ml}$ of casein solution for $2 \mathrm{~h}$ at room temperature. P-selectin site density on the polystyrene dishes was determined using timeresolved fluorometric detection of Eu-labeled streptavidin [16]. This protocol resulted in site densities of 7, 70 (interpolated) and 109 sites $/ \mu \mathrm{m}^{2}$ of P-selectin. As a negative control, some Pselectin-coated dishes were incubated with $1.0 \mu \mathrm{g}$ free $\mathrm{Rb} 40.34$ antibody for $30 \mathrm{~min}$ to block the antigen and washed 5 times with $0.05 \%$ Tween-20. Alternatively, some negative control dishes were incubated with casein alone.

\subsection{Laminar flow assay}

The inverted parallel plate flow chamber used in the in vitro microbubble adhesion efficiency experiments has been described earlier [16]. Dishes coated with P-selectin were secured to the flow chamber deck (GlycoTech, Rockville, MD) by vacuum suction, and the flow chamber was inverted by means of a specially fabricated holder. The flow rate was controlled by a withdrawal syringe pump. The inverted flow chamber was mounted on the stage of a Leitz Laborlux 11 microscope equipped with a $40 \times$ long working distance objective. Video microscopy data were recorded from a standard CCD camera operated at 30 frames/s onto standard VHS video tape or MiniDV digital video cassettes. An on-screen time stamp was achieved with a video timer.

Equal quantities of targeted wrinkled and spherical microbubbles were mixed and diluted to a concentration of $\sim 15 \times 10^{6} \mathrm{ml}^{-1}$ in $\mathrm{C}_{4} \mathrm{~F}_{10}$-saturated DPBS buffer. The microbubble dispersion was continuously mixed with a magnetic stir bar throughout each experiment to ensure constant flux of microbubbles through the flow chamber for the duration of the study.
A single field of view $(110 \mu \mathrm{m} \times 150 \mu \mathrm{m})$ close to the center of the flow deck was observed for the duration of each experiment. The flow chamber was observed under epifluorescent illumination and additional low-intensity bright-field transillumination, which enabled discrimination between fluorescently labeled wrinkled and unlabeled spherical microbubbles. Each dish was observed at a wall shear stress of $0.34,0.84$, or $1.3 \mathrm{dyn} / \mathrm{cm}^{2}$ for the duration of the experiment; a total flux of approximately 200 microbubbles of each population passing within the focal distance through the field of view was recorded for each dish. At least four dishes were observed for each experimental condition.

\subsection{Microbubble deformation}

Targeted wrinkled and spherical microbubbles were prepared separately as described above. Both populations were labeled with DiI in this experiment, which allowed visualization of deformation of the microbubble shell. A modified flow chamber was created to enable microscopy of adherent microbubbles at higher magnification, which was impossible in the standard flow chamber due to the thickness of the polystyrene dish. A rectangular polyvinylchloride coverslip $(2.2 \times 2.2 \times 0.028 \mathrm{~cm})$ (Electron Microscopy Sciences, Hatfield, PA), incubated overnight with $250 \mathrm{ng}$ P-selectin, was used as the target surface for these experiments, resulting in $117 \mu^{-2}$ receptor site surface density. A $1 \mathrm{~cm} \times 1 \mathrm{~cm}$ square hole was cut from the center of a standard $35 \mathrm{~mm}$ polystyrene dish, and the P-selectin coated coverslip was placed between the dish and flow chamber deck. The dish and coverslip were secured to the flow deck with suction. Wrinkled or spherical microbubbles were examined in separate flow chamber experiments.

Wrinkled or spherical microbubbles were bound to the target surface by infusion into the flow chamber at low wall shear stress $\left(0.24 \mathrm{dyn} / \mathrm{cm}^{2}\right)$. After $5 \mathrm{~min}$ of infusion, the microbubble dispersion was replaced with degassed DPBS so that no additional microbubbles were introduced. A minimum of 100 adherent microbubbles in five fields of view were examined. Still images were taken with a high-resolution digital camera (Orca, Hamamatsu, Shizuoka, Japan). The wall shear stress was sequentially increased to $0.40,1.3$ and $3.3 \mathrm{dyn} / \mathrm{cm}^{2}$ for $5 \mathrm{~min}$ each, and five fields of view were recorded at each wall shear stress. The camera was aligned such that the direction of flow was along the horizontal axis of the image. The deformation index of each adherent microbubble was calculated as the quotient of the maximal length of the microbubble in the direction of flow (L) and that of the perpendicular length (W). Microbubble deformation under zero flow was measured after pipetting microbubbles onto a P-selectin coated coverslip. Image pixels in this study were square, as checked with a stage micrometer aligned along the chamber length and width.

\subsection{Intravital microscopy}

Male C57Bl/6 $(N=4)$ (Hilltop Lab Animals, Scottsdale, PA) and P-selectin knockout $\left(\mathrm{P}^{-/-}\right)(N=3)$ mice (derived from the colony described in [37]) were used. All mice were between the ages of 8 and 12 weeks. Animal experimental protocols were 
approved by the institutional animal care and use committee. Pselectin expression in the cremaster muscle was induced by an intrascrotal injection of $0.5 \mu \mathrm{g}$ murine tumor necrosis factor alpha (TNF- $\alpha$ ) (Sigma, St. Louis, MO) 2 h before surgery. Mice were anesthetized with an intraperitoneal injection of $125 \mathrm{mg} / \mathrm{kg}$ body weight ketamine, $12.5 \mathrm{mg} / \mathrm{kg}$ body weight xylazine, and $0.025 \mathrm{mg} / \mathrm{kg}$ body weight atropine sulfate. Body temperature was maintained at $38^{\circ} \mathrm{C}$ during preparation with an electric heat pad. Trachea intubation to promote spontaneous respiration was accomplished with PE 90 tubing, and microbubbles were introduced into the right jugular vein through a PE 20 cannula. A cremaster muscle was exteriorized and continuously superfused with an isothermic bicarbonate-buffered solution as described [38]. The exteriorized cremaster was pinned to a custom-made microscope stage and placed beneath a saline immersion objective for intravital microscopy (Zeiss SW 40/0.8 NA). The preparation was visualized with a CCD camera (Hamamatsu 2400) and recorded on VHS videocassette for off-line analysis. Targeted DiI labeled wrinkled and targeted DiO labeled spherical microbubbles $\left(2.5 \times 10^{6}\right.$ each) were mixed in $150 \mu \mathrm{l}$ isotonic saline and injected through the jugular cannula, followed by a $25 \mu \mathrm{l}$ saline flush. Injection through the PE 20 cannula did not alter the size distribution of the microbubbles (data not shown). Microbubble retention in the cremaster microcirculation was assessed between 4 and 8 min after injection. Fluorescence microscopy was sequentially performed with filter sets for DiO and DiI to reveal the presence of adherent microbubbles of each type. The tissue was subsequently transilluminated with bright-field to locate the adherent microbubbles with respect to the vascular space. The centerline velocity of each vessel was determined using the photometric technique with a dual-slit photodiode (CircuSoft), and mean blood velocity $\left(V_{\mathrm{b}}\right)$ was calculated as described [39]. The wall shear stress $\left(\tau_{\mathrm{w}}\right)$ was estimated using a conversion factor of 4.9 that accounts for the bluntness of the velocity profile in venules [40]:

$\tau_{\mathrm{w}}=4.9 \eta\left(8 V_{\mathrm{b}}\right) / d$

where $\eta$ is the plasma viscosity and $d$ is the measured vessel diameter.

\subsection{Data analysis}

In vitro adhesion was determined off-line by counting the number of adherent wrinkled and spherical microbubbles. Microbubble adhesion was classified as either transient (adhesion persisting $<10 \mathrm{~s}$ ), or sustained (persisting $>10 \mathrm{~s}$ ). The cutoff between sustained and transient adhesion was determined from a pause-time histogram (see below). The number of adherent microbubbles was divided by the flux of microbubbles passing near the target surface to compute efficiency of adhesion. This parameter takes into account potential differences in the delivery of wrinkled and spherical microbubbles to the target surface. The adhesion efficiency of spherical and wrinkled microbubbles was compared at three wall shear stresses and three P-selectin site densities in the flow chamber. A paired sample $t$-test was performed to detect if the difference between the adhesion of the spherical and wrinkled microbubbles was statistically significant. For the intravital microscopy experiments, the number of adherent wrinkled and spherical microbubbles was determined in venules within 10 optical fields in each mouse. Significance of the hypothesis that wrinkled microbubble retention in vivo is greater than that of spherical was tested using the paired $t$-test. Microbubble size distributions were measured using a Coulter counter; histograms were produced with 256 bins at a bin size of $128 \mathrm{~nm}$. Percentile intervals were estimated within the resolution of the Coulter counter, and brackets on Coulter histograms represent the mid-fifty percentile intervals with error $\leq 3.0 \%$. Error bars on all figures represent the standard error of the mean (S.E.M.).

\section{Results}

\subsection{Production of wrinkles on targeted microbubbles}

Application of a positive hydrostatic pressure of $750 \mathrm{~mm} \mathrm{Hg}$ to a dispersion of spherical microbubbles resulted in the formation of outward-projected structures on the surface of the microbubbles that we describe generally as wrinkles. These wrinkles are evident in the fluorescent deconvolution micrographs (Fig. 1). Structural features were observed on the surface of the wrinkled microbubbles that may be described as folds (arrow, Fig. 1A) and arms
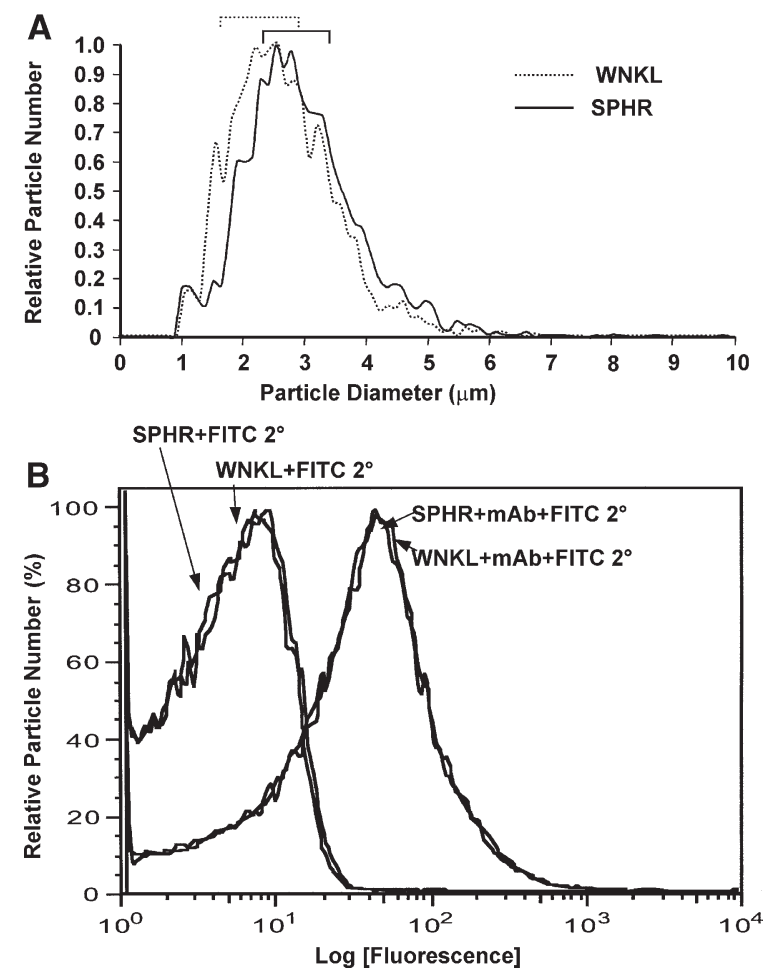

Fig. 2. Population characteristics of wrinkled and spherical microbubbles after conjugation to the targeting ligand Rb40.34. (A) Coulter counter size distributions of typical preparations of wrinkled (solid line) and spherical (dotted line) microbubbles. Mid-fifty percentile interval $(25-75 \%)$ is indicated by brackets. (B) Flow cytometry results confirming the presence of the targeting ligand on wrinkled and spherical microbubbles. The wrinkled and spherical histograms are nearly indistinguishable, and negative control data (SPHR + FITC $2^{\circ}$ and $\mathrm{WNKL}+$ FITC $2^{\circ}$ ) are plotted for both populations of microbubbles. 

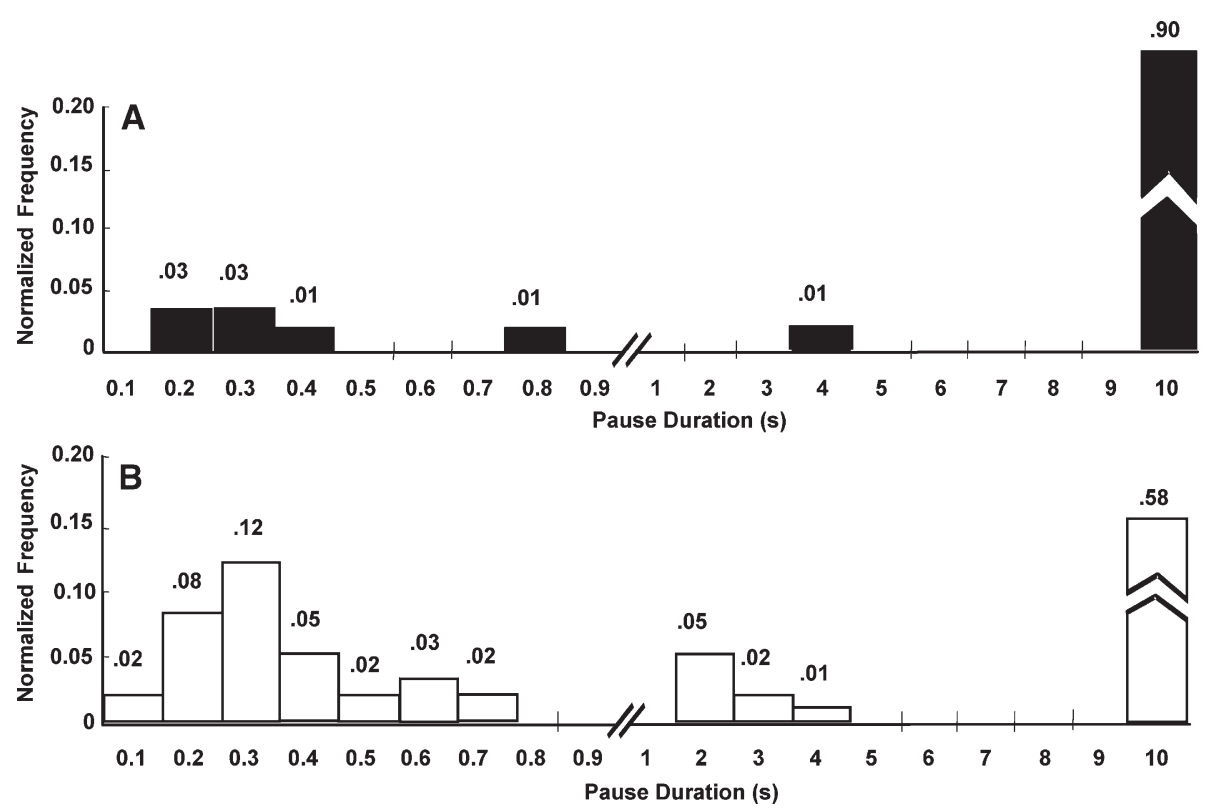

Fig. 3. Pause time histograms for (A) wrinkled and (B) spherical microbubbles on 109 sites $/ \mu^{2}$ P-selectin and at a wall shear stress of $0.84 \mathrm{dyn} / \mathrm{cm}^{2}$. Frequencies were normalized against the total number of adhesion events for each microbubble type; exact values of normalized frequency are printed at the top of each bin. 110 and 122 adhesive events were analyzed for wrinkled and spherical populations, respectively.

(arrow, Fig. 1B). The folds and arms were absent on the microbubbles that were not subjected to pressurization (Fig. 1C, D).

No visible change, such as re-inflation, was observed in the structure of the wrinkled microbubbles over several hours provided that the dispersion was kept in $\mathrm{C}_{4} \mathrm{~F}_{10}$-saturated DPBS (data not shown). Two mechanisms to account for these observations are possible. Pressurization of the microbubble may induce a loss of gas through the lipid shell, or the increased pressure may induce compression of the encapsulated gas. Both mechanisms result in a decrease of the internal volume of the microbubble gas core as well as the surface area of the gas-liquid interface. The resultant excess of the lipid shell surface area likely causes the formation of lipid folds, which are contiguous with the body of the microbubble and are observed to project outward. Potentially, these lipid folds may condense into bilayer structures and thus prevent re-inflation of the microbubble (due to gas influx or expansion) once the external pressure is released.

\subsection{Spherical and wrinkled microbubble characterization}

A Coulter counter was used to evaluate the size distributions of the wrinkled and spherical microbubbles. The wrinkled microbubble population is distinct from the parent spherical population in that the particle volume, but not necessarily the shell surface area, is reduced. The median diameter of the wrinkled microbubble preparation was $2.45 \mu \mathrm{m}$ and that of the parent spherical microbubble population was $2.94 \mu \mathrm{m}$ (Fig. 2A). The mid-fifty percentile (25-75\%) cutoffs were 1.80 and $2.96 \mu \mathrm{m}$ for the wrinkled population and 2.32 and $3.45 \mu \mathrm{m}$ for the spherical population. This difference in median microbubble diameter corresponds to a computed particle volume change of $42 \%$ and an excess surface area of $8.3 \mu \mathrm{m}^{2}(\sim 30 \%)$, assuming an initially spherical geometry.
The presence of the targeting ligand on the surface of the microbubbles was confirmed by flow cytometry (Fig. 2B). The wrinkled and spherical microbubbles possessed a nearly identical fluorescence intensity distribution, suggesting an equivalent surface density of the targeting ligand $\mathrm{Rb} 40.34$ on both
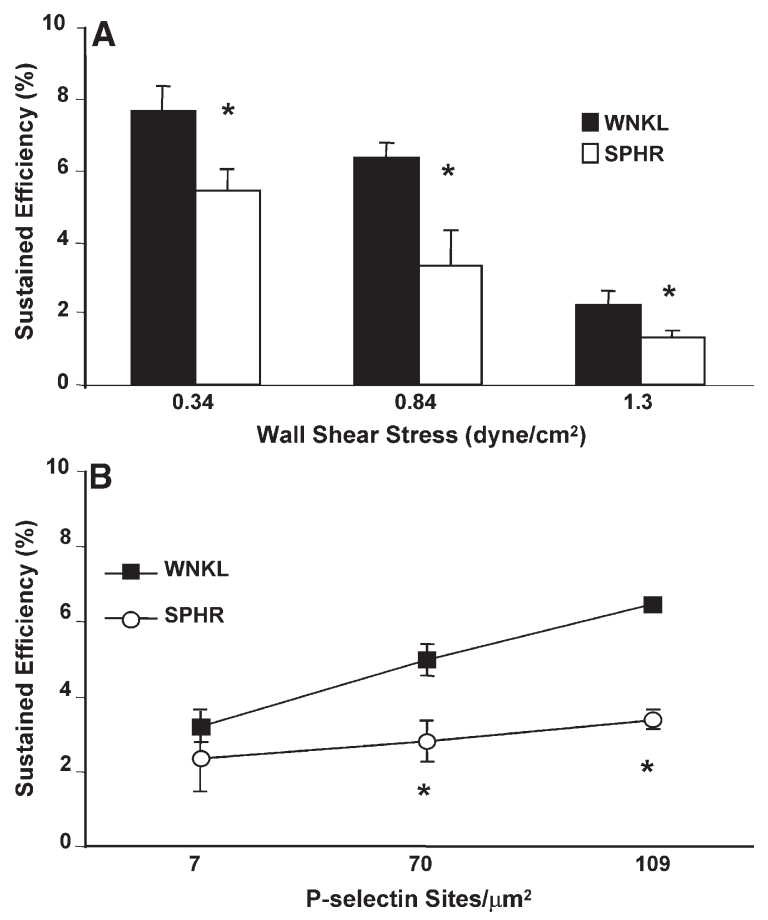

Fig. 4. Shear and site-density dependence of sustained attachment efficiency. (A) Sustained attachment efficiency of wrinkled and spherical microbubbles on 109 sites $/ \mu \mathrm{m}^{2} \mathrm{P}$-selectin under variable wall shear stress. (B) Sustained attachment efficiency at $0.84 \mathrm{dyn} / \mathrm{cm}^{2}$ on three P-selectin site densities; * $p<0.05$ between wrinkled and spherical populations. 
populations. Preliminary experiments suggest that the echogenicity of the wrinkled microbubble preparation is equivalent to that of the parent spherical population at clinically relevant imaging parameters. A thorough examination of wrinkled microbubble echogenicity will be presented in a subsequent publication.

\subsection{Pause time distributions of wrinkled and spherical microbubbles}

Pause times for wrinkled and spherical microbubbles were determined on P-selectin coated at 109 sites $/ \mu \mathrm{m}^{2}$ at $0.84 \mathrm{dyn} /$ $\mathrm{cm}^{2}$. These conditions were chosen to maximize the number of observable adhesive events for analysis, as fewer adhesive events were observed on lower site densities and higher wall shear stress (Fig. 5A, B). 110 or 122 adhesive events were examined at a temporal resolution of 30 frames/s. This analysis revealed two distinct types of attachment events that characterize the adhesion of wrinkled and spherical microbubbles. Wrinkled microbubbles showed transient adhesive events scattered at various durations, while spherical microbubbles appeared to form more frequent transient adhesion events with a mean duration of $0.3 \mathrm{~s}$. Our results showed that wrinkled microbubbles were more likely than spherical microbubbles to undergo sustained adhesion: $90 \%$ of adherent wrinkled microbubbles remained firmly bound for longer than 10 s (Fig. 3A), while only $58 \%$ of spherical microbubbles showed sustained adhesion (Fig. 3B). The majority of microbubbles that formed sustained adhesive events were observed to remain bound for much longer than $10 \mathrm{~s}$ (usually for the duration of the entire experiment). We observed relatively few microbubbles that remained adherent for between 1 and $10 \mathrm{~s}$ ( $1 \%$ for wrinkled and $8 \%$ for spherical): The majority of microbubbles formed adhesion events of duration greater than or equal to10 s, or less than $1 \mathrm{~s}$, which supported our assertion of two distinct types of adhesive events. We used these two types of adhesion to compare the attachment of wrinkled and spherical microbubbles. No intermediate adhesive events (such as rolling or skipping) were observed in the current experimental setup.

\subsection{Wrinkled microbubbles form sustained adhesion events with higher efficiency than spherical microbubbles in vitro}

The adhesion of P-selectin targeted microbubbles was examined in the flow chamber at wall shear stresses of $0.34,0.84$, and $1.3 \mathrm{dyn} / \mathrm{cm}^{2}$ on P-selectin site densities of 7, 70, and $109 \mu^{-2}$. Sustained adhesion of both wrinkled and spherical microbubbles was found to decrease with wall shear stress (Fig. 4A). Sustained adhesion was similarly dependent upon the substrate site density and increased as the P-selectin site density increased from 7 to 109 sites $/ \mu \mathrm{m}^{2}$ at $0.84 \mathrm{dyn} / \mathrm{cm}^{2}$ (Fig. 4B). At all of the conditions examined except the lowest site density $\left(7 \mathrm{sites} / \mu \mathrm{m}^{2}\right)$ the wrinkled microbubbles exhibited significantly $(p<0.05)$ greater sustained adhesion than spherical microbubbles.

To test whether enhanced adhesion could be due to the reduced size of the wrinkled microbubbles, we prepared spherical micro- bubbles with a size distribution essentially equal to that of the wrinkled population (median diameter difference $<1 \%$, Fig. $5 \mathrm{~B}$ ). We observed the same trend as described above, suggesting that the slightly smaller diameter of the typical wrinkled microbubbles does not contribute to their increased efficiency of adhesion (Fig. 5A). Targeting wrinkled or spherical microbubbles with an isotype control antibody (Iso $\mathrm{Ab}$ ), or infusing P-selectin-targeted microbubbles over a substrate of pure casein resulted in negligible sustained adhesion $(<2 \%)$ (Fig. 5C).

Microbubble capture efficiency was calculated as the combined efficiency of sustained and transient adhesion. This
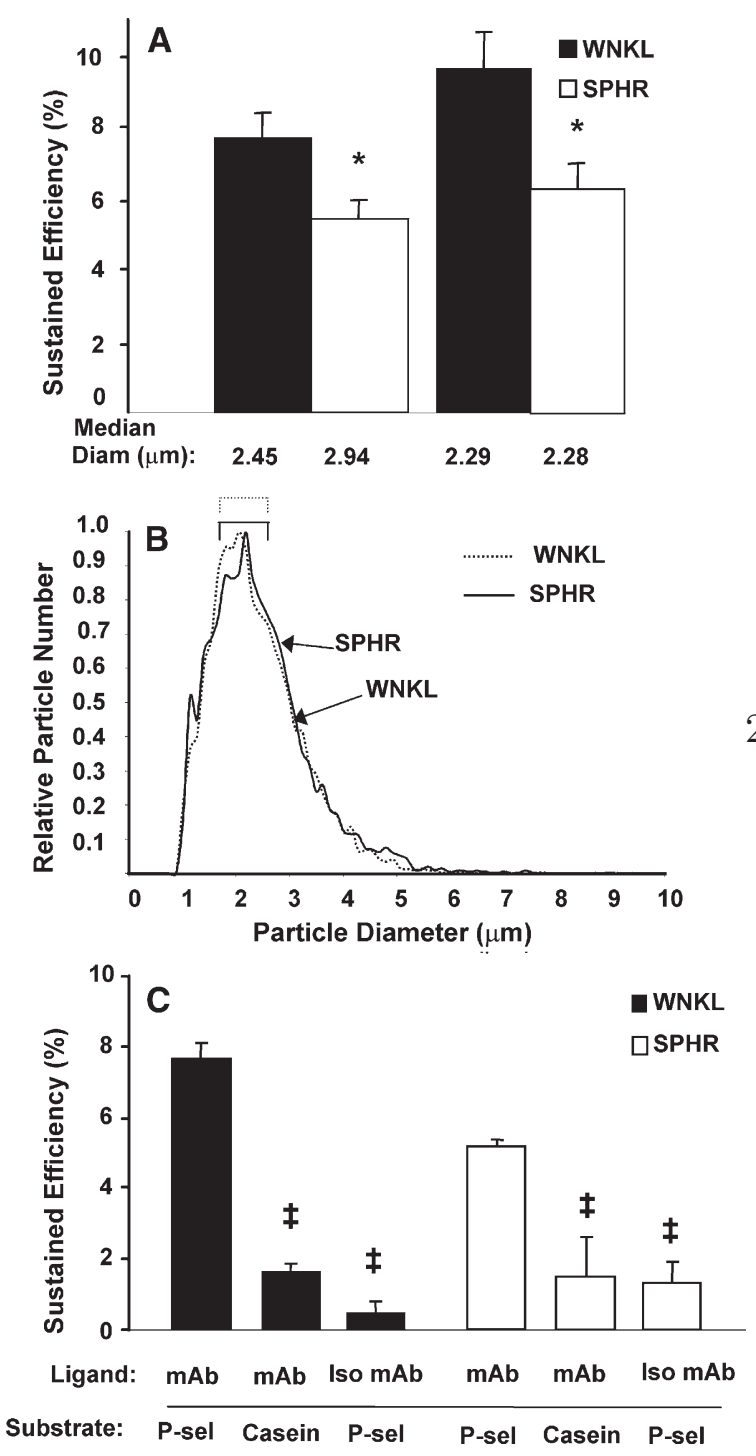

Fig. 5. Sustained attachment efficiency of targeted microbubbles. (A) Sustained attachment efficiency $\left(0.34 \mathrm{dyn} / \mathrm{cm}^{2}\right.$ on 109 sites $/ \mu \mathrm{m}^{2}$ P-selectin) of typical wrinkled and spherical microbubbles, which possess different diameters, and equal-diameter wrinkled and spherical microbubbles; $* p<0.05$ between wrinkled and spherical microbubbles. (B) Size distributions of equal-diameter wrinkled (solid line) and spherical (dotted line) microbubbles. The mid-fifty percentile bounds were 1.67 and $2.70 \mu \mathrm{m}$ ( 8 bins of Coulter counter distribution) for wrinkled and spherical microbubbles and are indicated by the brackets. (C) Sustained microbubble adhesion using negative control ligand or substrate at $0.34 \mathrm{dyn} / \mathrm{cm}^{2} .{ }^{\star} p<0.05$ between indicated condition and Rb40.34-targeted microbubbles on 109 sites $/ \mu \mathrm{m}^{2} \mathrm{P}$-selectin. 
represents the ability of a microbubble to be recruited to the substrate and undergo an adhesion event without a reference to whether this event is transient or sustained. Capture efficiency decreased with wall shear stress increase (Fig. 6A) and increased with site density increase (Fig. 6B). There was no significant difference in capture efficiency between the spherical and wrinkled microbubble preparations under any conditions.

The fraction of sustained adhesion events, which represents the likelihood that a captured microbubble forms a sustained (as opposed to a transient) adhesive event, was calculated by dividing the sustained adhesion efficiency by the capture efficiency. The sustained adhesion fraction was studied as a function of wall shear stress (Fig. 6C) and P-selectin site density (Fig. 6D). Under all conditions examined, the wrinkled microbubbles maintained a significantly greater sustained fraction than the spherical microbubbles. The sustained adhesion fraction for both spherical and wrinkled microbubbles appeared to be invariant over the range of wall shear stress and target site density examined. Therefore, the ability of a captured microbubble to preferentially achieve sustained adhesion might be applicable to a wide range of hydrodynamic and substrate conditions.

\subsection{Adherent wrinkled microbubbles deform under shear stress}

We hypothesized that the enhanced sustained adhesion of wrinkled microbubbles was due to an alteration in shape induced by the fluid force upon the adherent microbubble. Wall shear stress is a measure of this fluid force at the flow chamber boundary on a per area basis. To test this hypothesis, the deformability and orientation of wrinkled and spherical microbubbles under in- creasing wall shear stress was examined at high spatial resolution in the flow chamber. A deformation index above 1 indicates elongation and orientation in the direction of flow. The deformation index of the wrinkled microbubbles was significantly greater than that of the spherical microbubbles over the range of wall shear stresses $\left(0.34-1.3 \mathrm{dyn} / \mathrm{cm}^{2}\right)$ at which adhesion efficiency was examined (Fig. 7A). Deformation appeared to plateau at approximately $1.3 \mathrm{dyn} / \mathrm{cm}^{2}$.

To examine whether the observed increase in deformation index was due to actual deformation, rather than simply orientation in the direction of flow, we calculated the coefficient of variance. If increasing wall shear stress caused an increasing proportion of the adherent wrinkled microbubbles to become oriented with their long axis in the direction of flow, the population variance would be expected to be greatest at low wall shear stress, and decrease as wall shear stress increased. However, we observed the lowest variance at low wall shear stress, and population variance increased with increasing wall shear stress (Fig. 7B). Therefore, the increase of wrinkled microbubble deformation index is likely due to deformation caused by the incident fluid shear, rather than an artifact caused by reorientation.

\subsection{Wrinkled microbubbles exhibit enhanced adhesion in the inflamed mouse cremaster}

Adhesion of wrinkled and spherical microbubbles in vivo was examined in a mouse model of inflammation. The wrinkled microbubbles exhibited significantly greater adhesion than coinjected spherical microbubbles in venules of $4 \mathrm{TNF}-\alpha$ treated
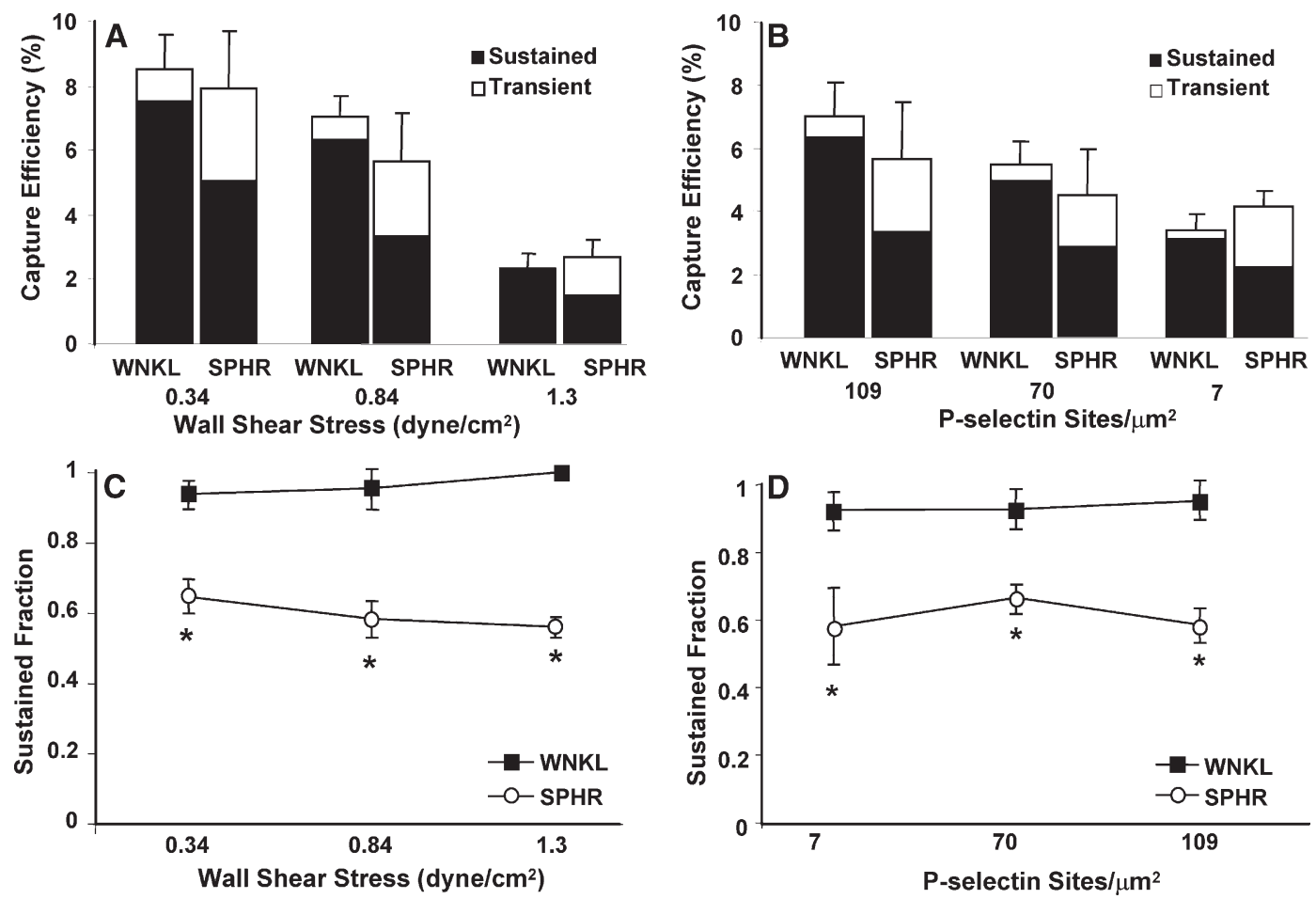

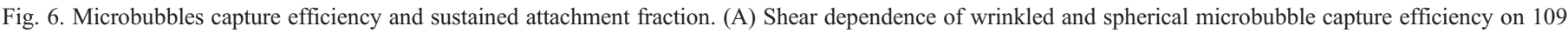

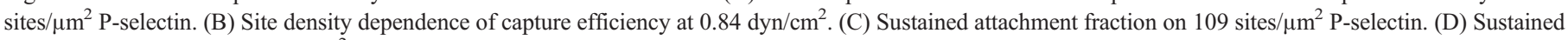
attachment fraction at $0.84 \mathrm{dyn} / \mathrm{cm}^{2} .{ }^{*} p<0.05$ between wrinkled and spherical microbubbles. 

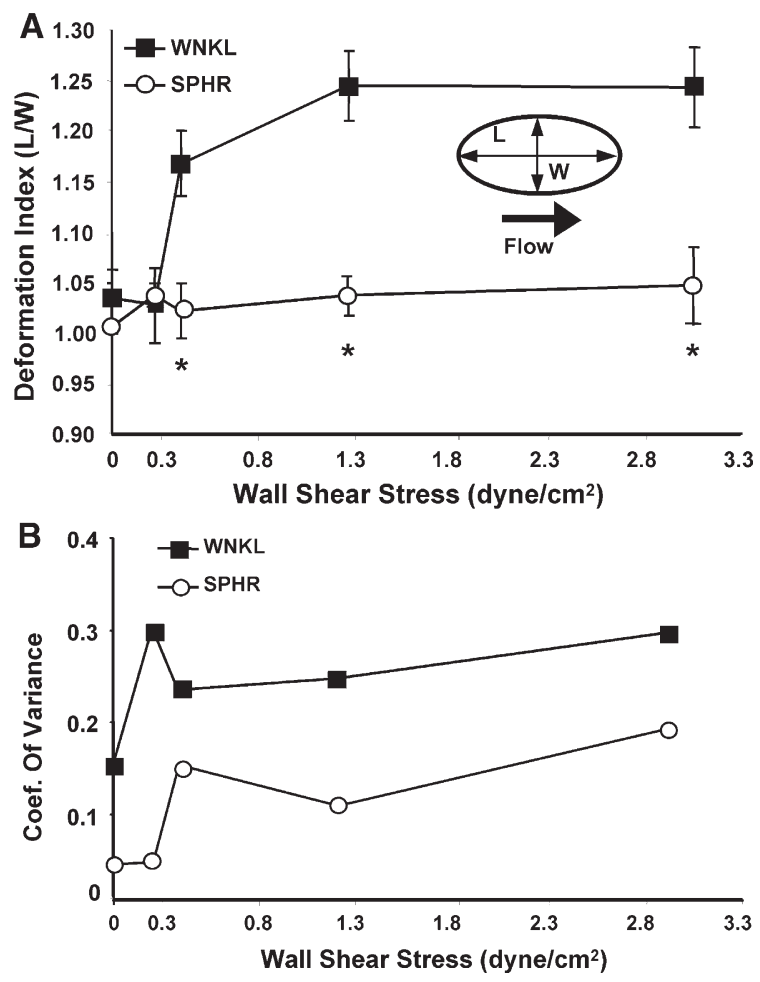

Fig. 7. Deformation index of wrinkled and spherical microbubbles under variable wall shear stress. (A) Deformation index was calculated as the degree of elongation in the direction of flow (L) divided by the axis length of the microbubble perpendicular to flow (W). ${ }^{*} p<0.05$ between spherical and wrinkled microbubbles. The coefficient of variance calculated for the deformation index of the wrinkled and spherical microbubbles is plotted in (B).

C57B1/6 mice (Fig. 8A). The adhesion of spherical targeted microbubbles in P-selectin deficient mice $\left(\mathrm{P}^{-/-}\right)$was reduced approximately three-fold, similar to results reported previously [3]; targeted adhesion of wrinkled microbubbles in $\mathrm{P}^{-/-}$mouse cremaster dropped nearly seven-fold. We observed no significant difference in adhesion between the wrinkled and spherical microbubbles in the $\mathrm{P}^{-1-}$ mice.

\section{Discussion}

Several factors control the efficacy of targeted particle adhesion. As a first step, efficient capture of free-stream microbubbles to the endothelial surface is necessary. Achieving a high signal-to-noise ratio of targeted ultrasound contrast requires the recruitment and retention of a sufficient fraction of the injected microbubbles on the intended target surface. Our data revealed that modifying the structure of the microbubble to enhance deformability does not alter the ability of an antibody-targeted microbubble near the surface to bind its target (a process defined here as capture). Via a mechanism that we hypothesized could be appropriated to enhance microbubble capture, microvilli-bearing clustered adhesion molecules [29,41,42] are responsible for efficient capture [43] of leukocytes to inflamed endothelium. The outward-projected structures on the surface of the wrinkled microbubbles resemble neutrophil microvilli, although the relatively scant [41] number of projections per microbubble (2-3) and lack of ligand clustering may be responsible for the observed capture efficiency. Additionally, it is possible that the kinetic properties of the targeting ligand (here, a monoclonal antibody), rather than the mechanical properties of the particle, are the chief determinant of capture efficiency.

Many antibodies, unlike physiological selectin ligands, are not inherently suited to rapid adhesion under high shear [44]. The ability of a ligand to achieve efficient adhesion in shear flow is related to both the kinetic and biomechanical properties of the molecule [26]. Previous studies [45,46] using antibodies as targeting ligands reported low levels of adhesion at even moderately increasing wall shear stress, similar to the results presented here. We found a maximum capture efficiency of $\sim 8 \%$ at $0.32 \mathrm{dyn} / \mathrm{cm}^{2}$ and 109 sites $/ \mu \mathrm{m}^{2}$, and capture of both wrinkled and spherical microbubbles decreased with wall shear stress. Capture efficiencies of non-deformable microbeads targeted with physiological capture ligands on purified selectin substrates are on the order of $\sim 10-35 \%[20,47]$ under similar conditions. Leukocytes roll on cytokine-stimulated endothelium with an efficiency of $\sim 20-30 \%$ in mouse venules [38], although the in vivo situation presents a substantially different target geometry and flow conditions. Although the wrinkled microbubbles described here achieved a degree of deformation similar to activated leukocytes $[18,43]$, our results suggest that deformation alone does not necessarily compensate for inefficient binding biomechanics. Another requirement for successful targeted adhesion is high efficiency of retention. It is necessary for an adherent microbubble to remain firmly bound to the target site: In the case of targeted contrast imaging,

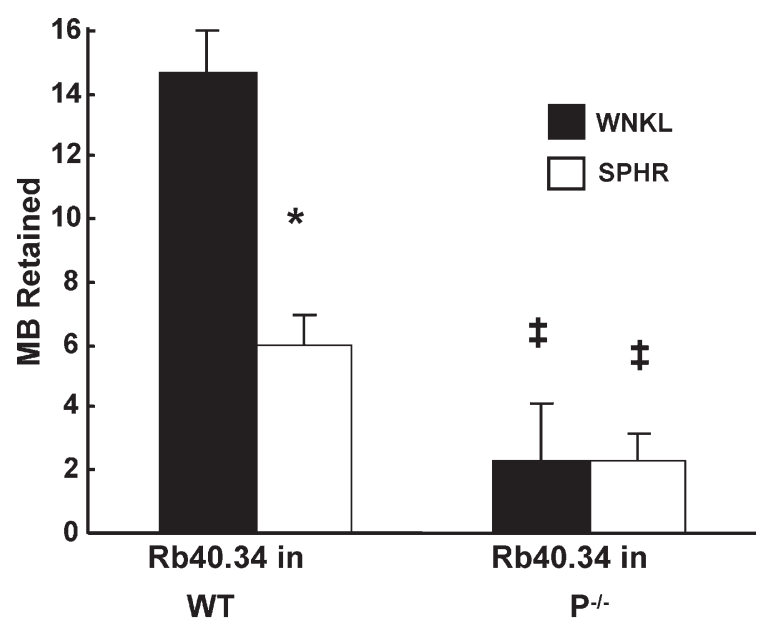

\begin{tabular}{c|c|c} 
& WT & $P^{-/-}$ \\
\hline Venule Diam. $(\mu \mathrm{m})$ & $43 \pm 9$ & $37 \pm 3$ \\
\hline$V_{b}(\mathrm{~mm} / \mathrm{s})$ & $2.9 \pm 0.4$ & $2.9 \pm 0.2$ \\
\hline WSS (dyne/cm $\left.{ }^{2}\right)$ & $33 \pm 8.0$ & $39 \pm 2.5$
\end{tabular}

Fig. 8. Wrinkled and spherical microbubble adhesion in the inflamed mouse cremaster. Wild-type $(N=4)$ and $\mathrm{P}^{-/}(N=3)$ mice were treated with $500 \mathrm{ng}$ murine TNF- $\alpha 2$ h before surgery. (A) Total retention of wrinkled and spherical microbubbles in 10 venules. (B) Physiological parameters. ${ }^{*} p<0.05$ between wrinkled and spherical microbubbles, and ${ }^{\ddagger} p<0.05$ between indicated population in $\mathrm{P}^{-/-}$and wild-type animals. 
ultrasound scanning of the target region is usually performed after the circulating microbubble population has cleared from the bloodstream [48], and adherent microbubbles must persist at the target while unbound microbubbles are cleared. We report that the antibody-bearing microbubbles used in this study exhibit two adhesive states, referred to as sustained and transient adhesion. Wrinkled microbubbles formed sustained attachment events more frequently than spherical microbubbles, and our results suggest that this behavior is related to the increased deformability of the wrinkled microbubbles. Although wrinkled and spherical microbubbles exhibit similar efficiencies of capture, our data show that wrinkled microbubbles are better able to convert capture events into sustained adhesion. We interpret the invariability of this property across the wall shear stresses and substrate site densities used in this study as evidence that wrinkled microbubbles are inherently able to achieve a more robust and stable adhesive state than spherical microbubbles.

We hypothesized that an increase in wall shear stress would result in an increased degree of wrinkled microbubble deformation, and a corresponding increase in bond number. Our observations supported the hypothesis of shear-dependent microbubble deformability, and we confirmed that spherical microbubbles are largely non-deformable in the shear flow range studied. Wrinkled microbubbles in solution or bound under static conditions appear to bear flat membranous projections (which are likely bilayer folds) devoid of gaseous volume; once such a microbubble is captured to the target surface, we assume that the fluid force on the adherent microbubble induces these projections to rejoin the bulk of the particle body. A contiguous contact area, bearing a large number of bonds, may thus be formed. However, the tethering bond or bond cluster must be maintained for sufficient time for this deformation to occur. Several models predict a significant inrease in bond dissociation with applied force $[49,50]$, and at increasing shear it is possible that the microbubble-target bond will be broken before rearrangement of the surface membrane occurs. Alternatively, the excess membrane may not form a flat contact area, but rather be withdrawn as a single tether anchored at the capture site. This mechanism has been hypothesized to stabilize leukocyte adhesion by transferring energy from breaking of the tethering bond to extending the microvilli $[28,29]$. Our observations of elongated microbubbles do not seem to support the possibility of tethers. However, each of these mechanisms assumes that the force required to rearrange or deform the excess microbubble shell is less than that required to break the tethering microbubble-target bond(s), and it is possible that both tether elongation and full deformation occur to some extent. Further studies of the mechanical stiffness of microbubble wrinkles and the force required for tether extraction are necessary to clarify the exact mechanism of action.

We hypothesized that increased microbubble deformation, and the corresponding increase in bond number, at high shear would result in enhanced sustained adhesion; however, we observed that the efficiency of adhesion decreased with wall shear stress. This suggests that factors other than the overall extent of deformation (presumably proportional to the number of adhesive bonds) may also influence the sustainability of adhesion. One aspect not addressed in this research is the loading of the antibody-P-selectin bond upon capture. It is possible that the outward-projected wrinkles reduce the force on the adhesive bond by increasing the lever arm between the center of the microbubble and the rearmost anchoring bond [26]. This effect is due to the geometry of the system and is independent of any assumed relationship between bond force and dissociation. A microbubble bound by a single antibody molecule located at the tip of a $0.5 \mu \mathrm{m}$ wrinkle experiences a $\sim 55 \%$ lower bond force than a spherical particle in which the ligand is flush with the microbubble surface. This may not necessarily result in an increase in capture, but rather in the stabilization of a captured particle. Indeed, our results indicated no change in wrinkled microbubble capture efficiency, although wrinkled microbubbles were better able to convert capture into sustained adhesion. The reduced loading may enable a captured microbubble to remain adherent long enough for additional stabilizing bonds to form, resulting in sustained, rather than transient, adhesion. Thus, it is possible that reduced loading due to lengthening of the lever arm, rather than deformation-induced increase in bond number, may underlay the observed improvement of wrinkled microbubble sustained adhesion. Further experiments with non-deformable microbubbles bearing outward-projected structures are necessary to clarify whether geometry or deformation are ultimately responsible for the enhanced sustained adhesion of wrinkled microbubbles.

\section{Conclusion}

A simple method of creating targeted microbubbles for contrast-enhanced ultrasound that bear projections and exhibit deformation in shear flow was devised. The adhesion of these wrinkled microbubbles tended to persist over a significantly longer time scale than that of spherical microbubbles, and wrinkled microbubbles were shown to deform within the shear regime at which enhanced sustained adhesion was observed. The wrinkled microbubbles maintained a relatively constant proportion of sustained, as opposed to transient, adhesive events over a wide range of wall shear stresses and target site densities, which suggests that deformability enables a more robust state of adhesion. This property may be of importance not only for targeted microbubble applications, but also for general drug delivery particle use, especially for targeting pathology in high-shear blood vessels.

\section{Acknowledgement}

This work was supported in part by NIH BRP HL 64381 and a research grant from the Whitaker Foundation to K. Ley. J.J. Rychak was supported via NIH Cardiovascular training grant HL07284 to UVA. Generous donation of laboratory equipment to A.L. Klibanov's laboratory at UVA Cardiovascular Division by Mallinckrodt Inc. (St. Louis, MO) is appreciated. A.L. Klibanov was supported in part via DOE 43-14702, NIH DK63508 and HL64381, and EIF grant to Cardiovascular Division. J.R. Lindner was supported in part via NIH DK63508, HL64381 and Grant-inAid GF10013 from the Atlantic Coast Affiliate of American Heart 
Association, Baltimore, MD. The authors are appreciative to C. Bachmann and M. Smith for their valuable discussion, to Prof. B. Helmke for performing deconvolution microscopy experiments, and to M. Solga for assistance with flow cytometry.

\section{References}

[1] J.R. Lindner, Microbubbles in medical imaging: current applications and future directions, Nat. Rev. Drug Discov. 3 (2004) 527-532.

[2] A.L. Klibanov, Ultrasound contrast agents: development of the field and current status, Top. Curr. Chem. 222 (2002) 73-106.

[3] J.R. Lindner, J. Song, J. Christiansen, A.L. Klibanov, F. Xu, K. Ley, Ultrasound assessment of inflammation and renal tissue injury with microbubbles targeted to P-selectin, Circulation 104 (2001) 2107-2112.

[4] F.S. Villanueva, R.J. Jankowski, S. Klibanov, M.L. Pina, S.M. Alber, S.C. Watkins, G.H. Brandenburger, W.R. Wagner, Microbubbles targeted to intracellular adhesion molecule-1 bind to activated coronary artery endothelial cells, Circulation 98 (1998) 1-5.

[5] G.E.R. Weller, E. Lu, M.M. Csikari, A.L. Klibanov, D. Fischer, W.R. Wagner, F.S. Villanueva, Ultrasound imaging of acute cardiac transplant rejection with microbubbles targeted to intercellular adhesion molecule-1, Circulation 108 (2003) 218-224.

[6] E.C. Unger, T.P. McCreery, R.H. Sweitzer, D. Shen, G. Wu, In vitro studies of a new thrombus-specific ultrasound contrast agent, Am. J. Cardiol. 81 (1998) 58G-61G.

[7] P.A. Schumann, J.P. Christiansen, R.M. Quigley, T.P. McCreery, R.H. Sweitzer, E.C. Unger, J.R. Lindner, T.O. Matsunaga, Targeted-microbubble binding selectively to GPIIb/IIIa receptors of platelet thrombi, Invest. Radiol. 37 (2002) 587-593.

[8] D.B. Ellegala, H. Leong-Poi, J.E. Carpenter, A.L. Klibanov, S. Kaul, M.E. Shaffrey, J. Sklenar, J.R. Lindner, Imaging tumor angiogenesis with contrast ultrasound and microbubbles targeted to alpha(v) beta3, Circulation 108 (2003) 336-341.

[9] H. Leong-Poi, J. Christiansen, A.L. Klibanov, S. Kaul, J.R. Lindner, Noninvasive assessment of angiogenesis by ultrasound and microbubbles targeted to alpha ${ }_{\mathrm{v}}$-integrins, Circulation 107 (2003) 455-460.

[10] G.E.R. Weller, M.K.K. Wong, R.A. Modzelewski, E. Lu, A.L. Klibanov, W.R. Wagner, F.S. Villanueva, Ultrasonic imaging of tumor angiogenesis using contrast microbubbles targeted via the tumor-binding peptide arginine-arginine-leucine, Cancer Res. 65 (2005) 533-539.

[11] A.L. Klibanov, P.T. Rasche, M.S. Hughes, J.K. Wojdyla, K.P. Galen, J.H Wible Jr., G.H. Brandenburger, Detection of individual microbubbles of ultrasound contrast agents: imaging of free-floating and targeted bubbles, Invest Radiol. 39 (2004) 187-195.

[12] S.Y. Ho, E. Barbarese, J.S. D'Arrigo, C. Smith-Slatas, R.H. Simon, Evaluation of lipid-coated microbubbles as a delivery vehicle for Taxol in brain tumor therapy, Neurosurgery 40 (1997) 1260-1268.

[13] A.F.H. Lum, M.A. Borden, P.A. Dayton, D.E. Kruse, S.I. Simon, K.W. Ferrara, Ultrasound radiation force enables targeted deposition of model drug carriers loaded on microbubbles, J. Control. Release 111 (2006) 128-134.

[14] J.P. Christiansen, B.A. French, A.L. Klibanov, S. Kaul, J.R. Lindner, Targeted tissue transfection with ultrasound destruction of plasmid-bearing cationic microbubbles, Ultrasound Med. Biol. 29 (2003) 1759-1767.

[15] P.A. Frenkel, S. Chen, T. Thai, R.V. Shohet, P.A. Grayburn, DNA-loaded albumin microbubbles enhance ultrasound-mediated transfection in vitro, Ultrasound Med. Biol. 28 (2002) 817-822.

[16] A.M. Takalkar, A.L. Klibanov, J.J. Rychak, J.R. Lindner, K. Ley, Binding and detachment dynamics of microbubbles targeted to P-selectin under controlled shear flow, J. Control Release 96 (2004) 473-482.

[17] E.C. Butcher, Leukocyte-endothelial cell recognition: three (or more) steps to specificity and diversity, Cell 67 (1991) 1033-1036.

[18] E.R. Damiano, J. Westheider, A. Tözeren, K. Ley, Variation in the velocity, deformation, and adhesion energy density of leukocytes rolling within venules, Circ. Res. 79 (1996) 1122-1130.

[19] K.D. Rinker, V. Prabhakar, G.A. Trusky, Effect of contact time and force on monocyte adhesion to vascular endothelium, Biophys. J. 80 (2001) $1722-1732$.
[20] M.L. Smith, M.J. Smith, M.B. Lawrence, K. Ley, Viscosity-independent velocity of neutrophils rolling on P-selectin in vitro or in vivo, Microcirculation 9 (2002) 523-536.

[21] A. Atherton, G.V.R. Born, Quantitative investigation of the adhesiveness of circulating polymorphonuclear leukocytes to blood vessel walls, J. Physiol. 222 (1972) 447-474.

[22] J. Cao, B. Donell, D.R. Deaver, M.B. Lawrence, C. Dong, In vitro side view imaging technique and analysis of human T-leukemic cell adhesion to ICAM-1 in shear flow, Microvasc. Res. 55 (1998) 124-137.

[23] J.C. Firrell, H.H. Lipowsky, Leukocyte margination an deformation in mesenteric venules of rat, Am. J. Physiol. 256 (1989) H1667-H1674.

[24] T. Yago, A. Leppänen, H. Qiu, W.D. Marcus, M.U. Nollert, C. Zhu, R.D. Cummings, R.P. McEver, Distinct molecular and cellular contributions to stabilizing selectin-mediated rolling under flow, J. Cell Biol. 158 (2002) 787-799.

[25] S.D. House, H.H. Lipowsky, In vivo determination of the force on leukocyte-endothelium adhesion in the mesenteric microvasculature of the cat, Circ. Res. 63 (1988) 658-668.

[26] R. Alon, D.A. Hammer, T.A. Springer, Lifetime of the P-selectincarbohydrate bond and its response to tensile force in hydrodynamic flow, Nature 374 (1995) 539-542.

[27] U.H. Von Andrian, S.R. Hasslen, R.D. Nelson, S.L. Erlandsen, E.C. Butcher, A central role for microvillous receptor presentation in leukocyte adhesion under flow, Cell 82 (1995) 989-999.

[28] J.H.P. Shao, Ting-Beall, R.M. Hochmuth, Static and dynamic lengths of neutrophil microvilli, Proc. Natl. Acad. Sci. U. S. A. 95 (1998) $6797-6802$.

[29] E.Y.H. Park, M.J. Smith, E.S. Stropp, K.R. Snapp, J.A. DiVietro, W.F. Walker, D.W. Schmidtke, S.L. Diamond, M.B. Lawrence, Comparison of PSGL-1 microbead and neutrophil rolling: microvillus elongation stabilizes P-selectin bond clusters, Biophys. J. 82 (2002) 1835-1847.

[30] R. Stahn, C. Grittner, R. Zeisig, U. Karsten, S.B. Felix, K. Wenzel, Sialyl Lewis(x)-liposomes as vehicles for site-directed, E-selectin-mediated drug transfer into activated endothelial cells, Cell Mol. Life Sci. 58 (2001) $141-147$.

[31] H.S. Sakhalkar, M.K. Dalal, A.K. Salem, R. Ansari, J. Fu, M.F. Kiani, D.T. Kurjiaka, J. Hanes, K.M. Shakesheff, D.J. Goetz, Leukocyteinspired biodegradable particles that selectively and avidly adhere to inflamed endothelium in vitro and in vivo, Proc. Natl. Acad. Sci. U. S. A. 100 (2003) 15895-15900.

[32] G.E.R. Weller, F.S. Villanueva, E.M. Tom, W.R. Wagner, Targeted ultrasound contrast agents: in vitro assessment of endothelial dysfunction and multi-targeting to ICAM-1 and Sialyl Lewis ${ }^{\mathrm{x}}$, Biotechnol. Bioeng. 92 (2005) 780-788.

[33] R. Bosse, D. Vestweber, Only simultaneous blocking of the L- and P-selectin completely inhibits neutrophil migration into mouse peritoneum, Eur. J. Immunol. 24 (1994) 3019-3024.

[34] N.M. Green, A spectrophotometric assay for avidin and biotin based on binding of dyes by avidin, Biochem. J. 94 (1965) 23c-24c.

[35] A.L. Klibanov, H. Gu, J.K. Wojkyla, J.H. Wible Jr., D.H. Kim, D. Needham, F.S. Villanueva, G.H. Brandenburger, Attachment of ligands to gas-filled microbubbles via PEG spacer and lipid residues anchored at the interface, Proceedings of the International Symposium on Controlled Release of Bioactive Materials, vol. 26, 1999, pp. 124-125.

[36] B.P. Helmke, R.P. Goldman, P.F. Davies, Rapid displacement of vimentin intermediate filaments in living endothelial cells exposed to flow, Circ. Res. 86 (2000) 745-752.

[37] D.C. Bullard, L. Qin, I. Lorenzo, W.M. Quinlin, N.A. Doyle, R. Bosse, D. Vestweber, C.M. Doerschuk, A.L. Beaudet, P-selectin/ICAM-1 double mutant mice: acute emigration of neutrophils into the peritoneum is completely absent but is normal in pulmonary alveoli, J. Clin. Invest. 95 (1995) 1782-1788.

[38] K. Ley, D.C. Bullard, M.L. Arbones, R. Bosse, D. Vestweber, T.F. Tedder, A.L. Beaudet, Sequential contribution of L- and P-selectin to leukocyte rolling in vivo, J. Exp. Med. 181 (1995) 669-675.

[39] H.H. Lipowsky, B.W. Zweifach, Application of the "two-slit" photometric technique to the measurement of microvascular volumetric flow rates, Microvasc. Res. 15 (1978) 93-101. 
[40] D.S. Long, M.L. Smith, A.R. Pries, K. Ley, E.R. Damiano, Microviscometry reveals reduced blood viscosity and altered shear rate and shear stress profiles in microvessels after hemodilution, Proc. Natl. Acad. Sci. U. S. A. 101 (2004) 10060-10065.

[41] X. Li, D.A. Steeber, M.L.K. Tang, M.A. Rarrar, R.M. Perlmutter, T.F. Tedder, Regulation of L-selectin-mediated rolling through receptor dimerization, J. Exp. Med. 188 (1998) 1385-1390.

[42] K.R. Snapp, R. Craig, M. Herron, R.D. Nelson, L.M. Stoolman, G.S. Kansas, Dimerization of P-selectin glycoprotein ligand-1 (PSGL-1) required for optimal recognition of P-selectin, J. Cell Biol. 142 (1998) 263-270.

[43] E.B. Finger, R.E. Bruehl, D.F. Bainton, T.A. Springer, A differential role for cell shape in neutrophil tethering and rolling on endothelial selectins under flow, J. Immunol. 157 (1996) 5085-5096.

[44] S. Chen, R. Alon, R.C. Fuhlbrigge, T.A. Springer, Rolling and transient tethering of leukocytes on antibodies reveal specializations of selectins, Proc. Natl. Acad. Sci. U. S. A. 94 (1997) 3172-3177.
[45] A.O. Eniola, D.A. Hammer, Artificial polymeric cells for targeted drug delivery, J. Control. Release 87 (2003) 15-22.

[46] J.B. Dickerson, J.E. Blackwell, J.J. Ou, V.R. Patil, D.J. Goetz, Limited adhesion of biodegradable microspheres to E- and P-selectin under flow, Biotechnol. Bioeng. 73 (2001) 500-509.

[47] V.R.S. Patil, C.J. Campbell, Y.H. Yun, S.M. Slack, D.J. Goetz, Particle diameter influences adhesion under flow, Biophys. J. 80 (2001) 1733-1743.

[48] J.R. Lindner, K. Wei, Contrast echocardiography, Curr. Probl. Cardiol. 27 (2002) 451-517.

[49] G.I. Bell, Models for the specific adhesion of cells to cells: a theoretical framework for adhesion mediated by reversible bonds between cell surface molecules, Science 200 (1978) 618-627.

[50] E. Evans, K. Ritchie, Dynamic strength of molecular bonds, Biophys. J. 72 (1997) 1541-1555. 